\title{
The Effect of Clock and Carrier Frequency Offsets on the Performance of a Direct-Sequence Spread- Spectrum Multiple-Access System
}

\author{
ELVINO S. SOUSA, MEMBER, IEEE
}

\begin{abstract}
We consider a direct-sequence spread-spectrum multipleaccess network. It is widely known that the probability of symbol error in such a network is dependent on the carrier and spreading code chip phases of the interfering terminals with respect to the signal of interest. As a result, for a given total interference power, the performance is worse for the case of one strong interferer when compared to the case of many weak interferers where the Gaussian assumption on the multiple-access interference holds. We assume a random spreading code model and show that if there is an offset on the carrier and spreading code frequencies of the interfering signals relative to the signal of interest, the above difference in performance between the cases of a set of balanced and a set of unbalanced interferers is drastically reduced. The result is that subject to such frequency offsets we may make the Gaussian assumption on the interference power regardless of whether it is composed of one strong interferer or many weak interferers of the same total power. In a network it may be desirable to actually force the offsets by putting a slight offset on the clock oscillators of the various transmitters. The performance parameter is the variation of the noise variance for a given expected value of the total interference power. We show that to achieve a small variation of the noise variance, the spreading code and carrier offset frequencies should be greater than the symbol rate.
\end{abstract}

\section{INTRODUCTION}

$\mathrm{W}$ E consider a direct-sequence spread-spectrum multiple-access system in a ground radio networking environment. A major task in the design and analysis of such a system is the modeling of the system at the various layers. One of the necessary models is that which abstracts the key parameters of the communication process to use at the network level. For the purpose of traffic analysis a characterization of a link is required in terms of macro states of the system involving parameters like the positions of the terminals and their transmission characteristics. It is typically desirable to obtain the probability of packet success given that a packet is transmitted and there is a set of active interfering transmissions of various power levels.

Many analysis of direct-sequence spread-spectrum multiple-access systems have been presented in the liter-

Manuscript received February 17, 1989; revised December 2, 1989. This work was supported by the Natural Sciences and Engineering Research Council of Canada under a URF grant. This paper was presented in part at the IEEE Pacific Rim Conference on Communications, Computers, and Signal Processing, Victoria, B.C., Canada, June 1-2, 1989

The author is with the Department of Electrical Engineering, University of Toronto, Toronto, Ont. M5S 1 A4, Canada.

IEEE Log Number 9034785. ature. In [1]-[3] the probability of symbol error is calculated. The approach in these and other papers has been to be as exact as possible while taking into account carrier phase, chip phase, autocorrelation of sequences, and the relative phase of the symbol transmission clocks of the different transmitters. In these analyses, expressions for the noise variance are given in terms of the cross-correlations of sequences in ar sequence set. Numerical results are difficult to compute, especially for the case of interferers with unequal powers. Due to the number of parameters and computation required in many cases, the results are not easily applicable at the network level where it is desirable to characterize the link using a smaller set of parameters. A different approach to multiple-access analysis of direct sequence which is less accurate but relies on a simpler set of parameters is that typified by the analysis in [4]. For example, in [4] the multiple-access interference is treated as having the same effect as white Gaussian noise with a power spectral density that is easily calculated from the powers of the interfering signals and the background thermal noise power. Still in other papers the approach has been to model the multiple-access interference as Gaussian noise and obtain the capacity or cutoff rate of the channel [5].

Our model for multiple-access interference in a DS/ BPSK system is now described. First, we realize that cross-correlation functions of spreading codes are too complex to work with for most sets of sequences. Even if we assume-sequence sets with relatively well-known crosscorrelation properties such as Gold sequences, the crosscorrelations are very difficult to compute if the different transmissions are not symbol synchronized [1]. As a result we adopt the random model for the spreading codes as in [2]. This random sequence model has also been implicitly used in many analysis of CDMA. Based on the random chip model the interference at the detector due to an interferer that is chip synchronized with the signal being received has a binomial type of distribution. For large processing gains (defined as the number of chips per transmitted symbol) this distribution may be approximated by a Gaussian distribution. In the case of no chip level synchronization the interference due to one interferer is equal to a linear combination of two random variables with binomial-like distributions. For a large pro- 
cessing gain each component of this sum can again be approximated by a Gaussian random variable, hence the sum can also be approximated by a Gaussian random variable. In either case, the variance of the random variable is dependent on the carrier and chip phases of the interferer relative to those of the signal. In the case of multiple interfering signals the total interference is then a sum of random variables and is also Gaussian distributed with a variance equal to the sum of the individual variances. However, the individual variances are dependent on the carrier and spreading code phases and therefore are also random. For a set of symbols consituting a packet the probability density for the interference at the detector is Gaussian when conditioned on the variance. In [6], we computed the probability distribution function for the conditional variance and expressed the overall probability density function as the following expectation, or compound Gaussian probability density function

$$
f(\underline{x})=\int_{0}^{\infty} \frac{1}{(2 \pi v)^{n / 2}} \exp \left(\frac{1}{2 v}\|\underline{x}\|^{2}\right) f_{V}(v) d v
$$

where $f_{V}(\cdot)$ is the probability density for the conditional variance of the compound Gaussian process, and $n$ is the packet length (see [7], for a discussion of general properties of these processes). In [6], it was shown that the packet error probability is dependent on the density function $f_{V}(\cdot)$. For a given expected value $v_{0}=\boldsymbol{E}(V)$ the probability of error curve is closest to an ideal Shannon limit error curve for the case that $f_{V}(v)=\delta\left(v-v_{0}\right)$. Also in [6] we showed that for a given expected value of the total interference power the density function $f_{V}(\cdot)$ is dependent on the composition of the total interference power, hence the probability of packet success is also dependent on the composition of the total interference power. For the case of a large number of interferers of roughly equal power (a balanced set of interferers) the probability density approaches a delta function. For the case of an unbalanced power composition, the probability function $f_{V}(\cdot)$ will be broader. In other words, the variance of the total interference power at the detector over a set of symbols constituting a packet is a random variable. For a given expected value of this variance the performance is better if the variance has a small "variance." The worst case is that of a single interferer where the probability density function of the noise variance over one symbol is relatively broad as can be seen by examining the corresponding probability distribution function which we show in Fig. 1 along with the probability distribution function for the interference in the case of many weak interferers, or Gaussian noise.

The threshold model of the communication process that is desirable to include in a network model is based on three assumptions: 1) the multiple-access interference can be modeled as a Gaussian variable, i.e., at the detector the noise component is Gaussian; 2) the variance of the multiple-access interference is a simple function of the powers of the interfering signals; and 3) there is a high

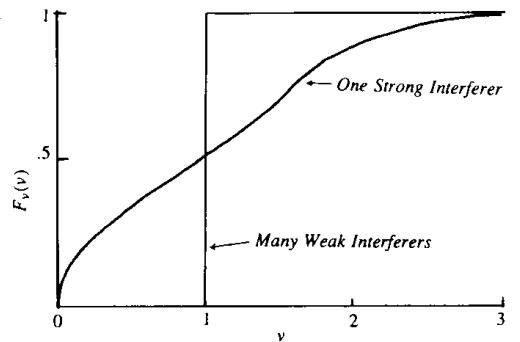

Fig. 1. Cumulative distribution functions for the normalized conditional variance of the interference for the cases of one strong interferer and many weak interferers.

degree of forward error correction so that a threshold model (predicted by Shannon's theorem) is applicable. The accuracy of the first assumption is dependent on the processing gain. For large processing gains the assumption can be justified by the Central Limit Theorem in the sense that a binomial distribution can be approximated by a Gaussian distribution. In any case, for a network where there is no synchronization at the symbol level, it is very difficult to use nonstatistical methods to determine the interference. The second assumption is dependent on the spread of the function $f_{V}(\cdot)$ being small; ideally the density should be a delta function and the detector noise is Gaussian. A constant detector noise variance for a given total interference power results in independent symbol errors within a packet and, for good codes with appropriate rates, an ideal (Shannon limit) error-performance curve. If the variance is random then the symbol errors within a packet are dependent and the limiting probability of error will not approach the Shannon limit for any coding scheme.

We would like to replace the above compound Gaussian process by a Gaussian process with variance equal to the expected value of the conditional variance. For a set of interferers and indeed for a given total interference power, the variance of the noise affecting the symbols of a packet should be constant from packet to packet and not dependent on the carrier and spreading code phases which are random from packet to packet. In other words, we would like the probability density for $f_{V}(\cdot)$ to be a delta function. We will see that the spread in the density $f_{V}(\cdot)$ may be decreased if we impose an offset on the carrier and spreading code frequencies of the interfering signals relative to the signal of interest. The result will be that an interference composition consisting of one strong interferer will have approximately the same probability of error characteristics as a composition consisting of many signals of approximately equal powers if the total interference power is the same in both cases. The probability of error curve can then be made as close to ideal as desirable by increasing the complexity of the error correcting code. Since the case of one strong interferer is the case where $f_{V}(\cdot)$ has the largest spread, in this paper we assume the case of one interferer and discuss ways to decrease the spread of $f_{V}(\cdot)$. If we modify the basic DS/ BPSK CDMA scheme to achieve this then we will im- 
prove performance in general and at the same time for well-coded systems the threshold model will apply.

From the above and as concluded in [6], the performance of DS/BPSK CDMA is solely dependent on the density function $f_{V}(\cdot)$. The exact form of the dependence will vary according to the coding scheme. We will not be more precise than to say that the performance depends on the "spread" of the density. One measure of the spread is the variance. Another good measure that relies on the density dropping to zero not far from the mean (i.e., no long tails) is the difference between the maximum ( $\max$ ) and minimum ( $\mathrm{min}$ ) values of the random variable where the density is nonzero. We refer to the interval ( $\min$-max) as the variation of the random variable. Due to computational considerations our performance criterion for an offset scheme that we will introduce is the variation.

\section{One Strong Interferer with Frequency OFFSETS}

We consider the case of a receiver that is attempting to receive the signal $x_{1}(t)$ in the presence of the strong interfering signal $x_{2}(t)$. Since our results will not depend on the actual power of the interfering signal we assume it to have the same power as the signal. The two signals are then given by

$$
x_{1}(t)=c_{1}(t) \cos \left(\omega_{1} t+\theta_{1}\right)
$$

and

$$
x_{2}(t)=c_{2}(t-\Delta) \cos \left(\omega_{2} t+\theta_{2}\right)
$$

where $c_{1}(t)$ and $c_{2}(t)$ are the spreading codes of the two signals modulated by the data which we assume is done digitally (i.e., data transitions occur at chip transitions), $\omega_{1}$ and $\omega_{2}$ are the carrier frequencies and $\theta_{1}$ and $\theta_{2}$ are the carrier phases, and $\Delta$ is the chip phase of the interfering signal with respect to that of the signal, as shown in Fig. 2 where we assume $t_{0}=0$. We assume that the chip periods of the spreading codes of the two signals have been normalized so that the period is 1 for $c_{1}(t)$ and $1+\tau$ for $c_{2}(t)$ where $\tau \ll 1$. The difference in carrier frequencies in hertz is denoted as $\nu=\left(\omega_{2}-\omega_{1}\right) / 2 \pi$. The spreading code is modeled as a Bernoulli process with values equal to \pm 1 . The clock rate of the spreading codes is equal to $N$ times the data rate. We also introduce the parameters $\alpha$ $=N \tau$, and $\gamma=N \nu$. The parameter $\alpha$ is the total chip phase difference between the two spreading codes accumulated over one data symbol period and can be shown to be approximately equal to the offset clock frequency normalized by the data rate for $\tau<<1$. The parameter $\gamma$ is the total carrier phase difference between the two signals accumulated over one data symbol, and can be shown to be equal to the offset frequency between the two carriers normalized by the data rate.

We assume a conventional correlator receiver. Up to a multiplicative constant the noise at the detector is given by

$$
I=2 \int_{0}^{N} x_{1}(t) x_{2}(t) d t
$$

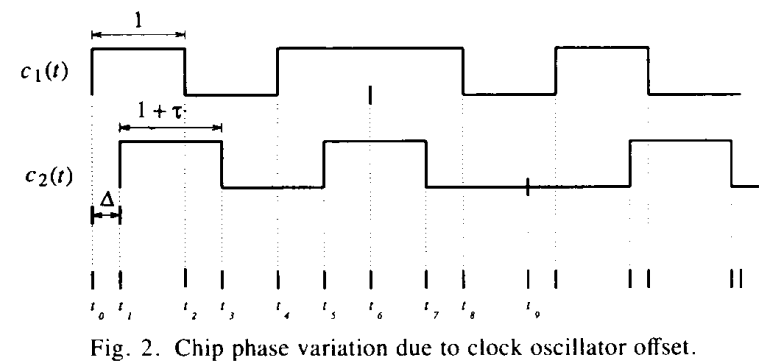

Referring to Fig. 2 we may write the above (after neglecting the integral of the double frequency term) as

$$
I=\sum_{i=0}^{2 N-1} \mu_{i} \int_{t_{i}}^{t_{i+1}} \cos (2 \pi \nu t+\theta) d t
$$

where $\theta=\theta_{2}-\theta_{1}$, the $t_{i}$ 's are the points at which the product of the two spreading codes changes value, and $\left\{\mu_{i}\right\}=\left\{c_{1,0} c_{2,0}, c_{1,0} c_{2,1}, c_{1,1} c_{2,1}, c_{1,1} c_{2,2}, \cdots\right\}$. The sequences $\left\{c_{1, i}\right\}$ and $\left\{c_{2, i}\right\}$ are Bernoulli sequences corresponding to the two spreading codes $c_{1}(t)$ and $c_{2}(t)$. From the above, we note that the $\mu_{i}$ 's are uncorrelated (actually also pairwise independent). Due to the small difference in the clock frequencies of the spreading codes the sequence of intervals $\left[t_{i}, t_{i+1}\right]$ can be thought of as consisting of two interleaved sequences. The length of the intervals in each sequence changes according to a triangular waveform and the waveforms corresponding to each subsequence are out of phase by half a period of the waveform [see Fig. 3(b)]. The period of the waveform is inversely proportional to $\tau$.

We are interested in systems with a symbol error probability greater than $10^{-4}$. Thus, for moderate to large processing gains, we may assume that the noise at the detector is Gaussian with zero mean (since $\boldsymbol{E}(I)=0$ ). Thus, it remains to calculate the variance which we obtain as a normalized value, after dividing by $N$, and denote by $V$. Since the variables $\mu_{i}$ and $\theta$ are dependent and the terms in the sum of (4) are uncorrelated we have

$$
\begin{aligned}
V & \triangleq \frac{1}{N} \boldsymbol{E}\left(I^{2}\right)=\frac{1}{N} \sum_{i=0}^{2 N-1} \boldsymbol{E}\left(\int_{t_{i}}^{t_{i+1}} \cos (2 \pi \nu t+\theta) d t\right)^{2} \\
& =\frac{1}{N} \sum_{i=0}^{2 N-1}\left(\frac{\sin \left(2 \pi \nu t_{i+1}+\theta\right)-\sin \left(2 \pi \nu t_{i}+\theta\right)}{2 \pi \nu}\right)^{2} .
\end{aligned}
$$

For large $N$ and $\nu \ll 1$ the difference of the two sine terms in (5) may be approximated by the product of the derivative of $\sin (2 \pi \nu t+\theta)$ and a time increment to obtain

$$
V=\frac{1}{N} \sum_{i=0}^{2 N-1}\left(t_{i+1}-t_{i}\right)^{2} \cos ^{2}\left(2 \pi v t_{i}+\theta\right) .
$$

We now use the functions in Fig. 3(a)-(b) to approximate the two subsequences of interval lengths and approximate the above sum with an integral on the interval 


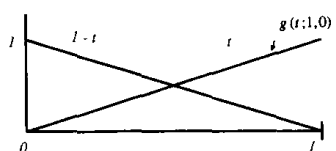

(a)
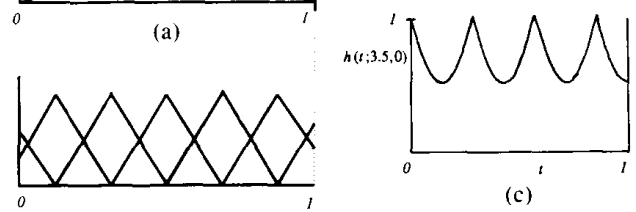

(c)

(b)

Fig. 3. The functions $g(t ; \alpha, \Delta)$ and $h(t ; \alpha, \Delta)$ : (a) $g(t ; \alpha, \Delta)$ and $1-$ $g(t ; \alpha, \Delta)$ for the case $\alpha=1$ and $\Delta=0 ;$ (b) $g(t ; \alpha, \Delta)$ and $1-g(t ;$ $\alpha, \Delta)$ for the case $\alpha>1$ and $\Delta \neq 0$; and (c) $h(t ; 3.5,0)$.

$[0,1]$. Expressing the dependence of $V$ on the actual set of parameters we obtain

$$
V(\alpha ; \gamma, \Delta, \theta)=\int_{0}^{1} h(t ; \alpha, \Delta) \cos ^{2}(2 \pi \gamma t+\theta) d t
$$

where

$$
h(t ; \alpha, \Delta)=g^{2}(t ; \alpha, \Delta)+[1-g(t ; \alpha, \Delta)]^{2}
$$

and $g(t ; \alpha, \Delta)$ is a triangular wave function defined as follows: the range is $[0,1]$, the period is $2 / \alpha, g(0)=$ $\Delta$, and $g(t)$ is increasing at $t=0$. Two examples of the function $g(t ; \alpha, \Delta)$ are shown in Fig. 3(a)-(b), and an example of the function $h(t ; \alpha, \Delta)$ is shown in Fig. 3(c). The function $h$ is periodic with period equal to $1 / \alpha$, and phase equal to $\Delta$. The phase takes values in $[0,1]$, and zero phase is the phase which makes $h(t ; \alpha, \Delta)$ attain its maximum value at $t=0$.

Equation (7) contains four parameters that determine the noise variance: $\alpha, \gamma, \Delta$, and $\theta$. The first two are the normalized spreading code and carrier offset frequencies and the last two are the spreading code chip phase and carrier phase at the start of a symbol transmission interval. We will view the variables $\alpha$ and $\gamma$ as deterministic and the variables $\Delta$ and $\theta$ as random. Since the last two variables are random, the variance is a random variable. For the case of zero carrier and chip frequency offsets (7) becomes

$$
V=\left[\Delta^{2}+(1-\Delta)^{2}\right] \cos ^{2} \theta .
$$

In this case the variance of the noise may be anywhere in the range $[0,1]$. The parameter $\theta$ determines the quantity of noise on the quadrature carrier; for $\theta=0$ all of the noise is in phase with the signal and for $\theta=\pi / 2$ all of the noise is out of phase. Similarly, the parameter $\Delta$ determines the variance of the correlation noise between the two spreading codes. The best case is that where the spreading code clocks are out of phase by $1 / 2$ of a chip where the noise variance is multipled by the factor $1 / 2$. The worst case is that where the two spreading codes are in phase where the factor is 1 . Assuming that $\theta$ is uniformly distributed in $[-\pi, \pi]$, and $\Delta$ is uniformly dis-

tributed in $[0,1]$ the expected value of $V$ for the case of zero frequency offsets is the well known result, $1 / 3$.

We now consider the case where there is an offset in clock and carrier frequencies. In this case the code and carrier phases vary at a rate proportional to the offset frequencies. The rates are $\nu$ (carrier) and approximately $\tau$ (spreading code, $\tau$ small) per chip time; or $\gamma$ (carrier) and $\alpha$ (spreading code) per symbol period. If the offset frequencies are equal to $1 / N$ then the resulting change in the phase over a symbol transmission interval is $2 \pi$ for the carrier and 1 for the spreading code. In this case, the variance should be approximately equal to the above expected value or $1 / 3$. Such a predictable value for the variance is preferable to the case of a random variance. We can thus conclude that clock and carrier frequency offsets are beneficial in the sense that they reduce the unpredictability of the variance at the detector. An offset in the frequencies of the spreading codes and carriers will result in the noise being due to a sum of correlations over small intervals with all of the possible phases, resulting in a value for the variance equal to the expected value. In a sense we have a situation where time averages equal ensemble averages.

Our task in this paper is to determine the variation in the noise variance, from the maximum variation of $(0-$ 1 ) in the case of zero frequency offsets to the case of zero variation where the variance is always equal to its expected value of $1 / 3$, as a function of the normalized frequency offsets $\alpha$ and $\gamma$. To obtain a constant noise variance we will see that the normalized spreading code offset frequency should be equal to an integer (if the carrier offset frequency is zero), and the normalized carrier offset frequency should be equal to an integer multiple of $1 / 2$ (if the spreading code offset frequency is zero). In these cases the phase changes, at a constant rate, by one period (one chip time or half of a carrier cycle time) during a symbol transmission time and the resultant noise is independent of the phases $\Delta$ and $\theta$ at the start of symbol transmission. If we have joint frequency offsets in the carrier and spreading code then there is an interaction between the two phases which makes it difficult to obtain a constant variance. The variation can however be made small so that it may be practically considered to be constant and close to $1 / 3$ which is the expected value of (8).

If the normalized offset frequencies are much less than unity, then the noise variance at the detection time of a symbol is strongly dependent on the value of the starting phases $\Delta$ and $\theta$. On the other hand, if the normalized offsets are not integers but greater than unity, then the variance is dependent on the starting phase to an extent that depends on the offset frequencies. The variance as a function of the offset frequency contains a set of sidelobes resembling a "sinc" function.

\section{Numerical Results}

To give some examples we compute the noise variance by summing (5) directly. As a consequence of the central limit theorem and also as verified by computation, the 


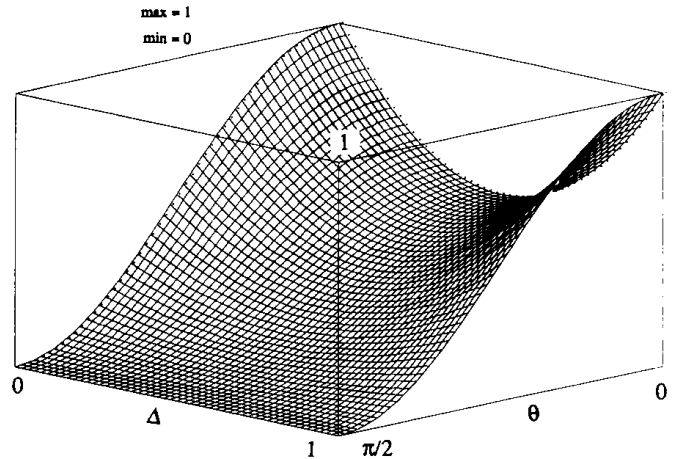

Fig. 4. Noise variance as a function of the starting spreading code phase $\Delta$ and the carrier phase $\theta$ in the case of zero frequency offsets ( $\alpha=0$, and $\gamma=0$ ).

computation of the variance using (5) is not too sensitive to $N$ for large $N$ and as a function of $N$ converges to a constant value. We use the value $N=100$ in computing some examples. Our goal is to study the variation of the noise variance as a function of the frequency offsets. For small frequency offsets the variance depends greatly on the initial phases $\theta$ and $\Delta$. As the frequency offsets are increased the dependence of the variance on the starting phase is weakened to the point that the variance is a constant for any starting phases. In Figs. 4-9 we show some three dimensional plots of the noise variance as a function of the starting phases $\Delta$ and $\theta$ parameterized by the normalized offset frequencies $\alpha$ and $\gamma$. In Fig. 4, we show the case of zero frequency offsets $\alpha=0$ and $\gamma=0$, hence the case of greatest variation. As a result of symmetry it is sufficient to show the variance for the ranges $0 \leq \Delta<$ 1 and $0<\theta<\pi / 2$. As shown in the figure we have for the pair $(\Delta, \theta): 0$ for $(\Delta, \pi / 2), 1$ for $(0,0)$, and $1 / 2$ for $(1 / 2,0)$. Fig. 5 shows the case where $\gamma=1$ and $\alpha$ $=0$. Since $\gamma$ is a multiple of $1 / 2$ and $\alpha=0$ the variance does not depend on the starting phase $\theta$ and the result corresponds to taking the expectation of (8) over the random variable $\theta$. Fig. 6 shows the opposite case of $\alpha=1$ and $\gamma=0$. In this case there is no dependence on the starting chip phase $\Delta$. The result corresponds to taking the expectation over $\Delta$ in (8). In Fig. 7, we show the case $\alpha=1$ and $\gamma=0.5$ where the variation in the variance is $(0.268$ 0.402 ). The opposite case $\alpha=0.5$ and $\gamma=1$ also has a similar variation (0.283-0.393) and is shown in Fig. 8. Fig. 9 shows the case $\alpha=1$ and $\gamma=1$. In this case the variation is small and equal to (0.321-0.356).

\section{A. Zero Carrier Frequency Offset}

We now look at some special cases of (5). We may obtain a closed-form approximation for the sum in (5) in the case of a zero carrier frequency offset by working with the integral approximation given in (7). In this case since the dependence on $\theta$ is well understood (i.e., a multiplication by $\cos ^{2} \theta$ ) we also assume $\theta=0$ in the following. For $\gamma=0$ and $\theta=0$ the normalized variance is

$$
V(\alpha, 0 ; \Delta, 0)=\int_{0}^{l} h(t ; \alpha, \Delta) d t .
$$

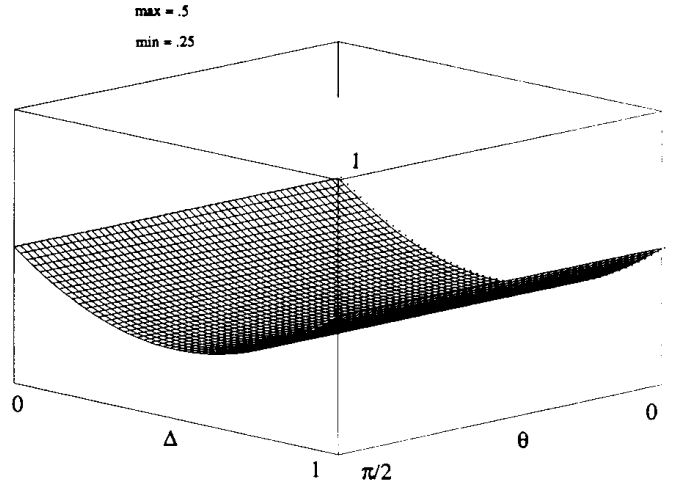

Fig. 5. Noise variance as a function of the starting spreading code phase $\Delta$ and the carrier phase $\theta$ for normalized frequency offsets $\alpha=0$, and $\gamma$ $=1$.

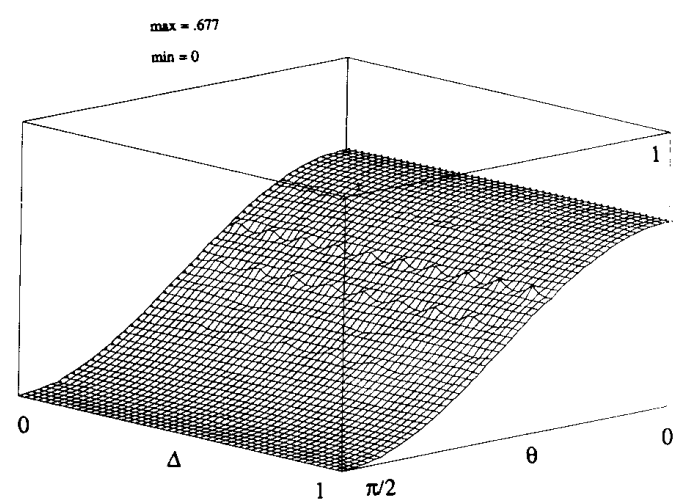

Fig. 6. Noise variance as a function of the starting spreading code phase $\Delta$ and the carrier phase $\theta$ for normalized frequency offsets $\alpha=1$, and $\gamma$ $=0$

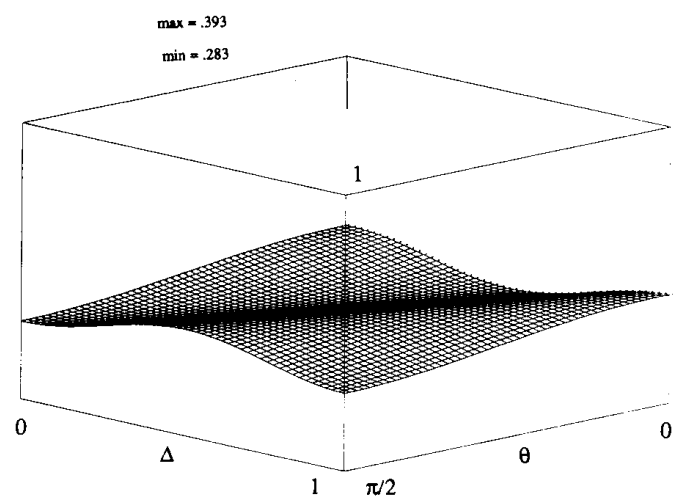

Fig. 7. Noise variance as a function of the starting spreading code phase $\Delta$ and the carrier phase $\theta$ for normalized frequency offsets $\alpha=1$, and $\gamma$ $=0.5$.

As an example if $\Delta=0$ and $\alpha=1$ the above integral becomes

$$
V(1,0 ; 0,0)=\int_{0}^{1}\left[t^{2}+(1-t)^{2}\right] d t .
$$

This integral has the value $2 / 3$ which is equal to the expected value of the variance in (8) if we set $\theta=0$.

To give the result for the general case we define the 


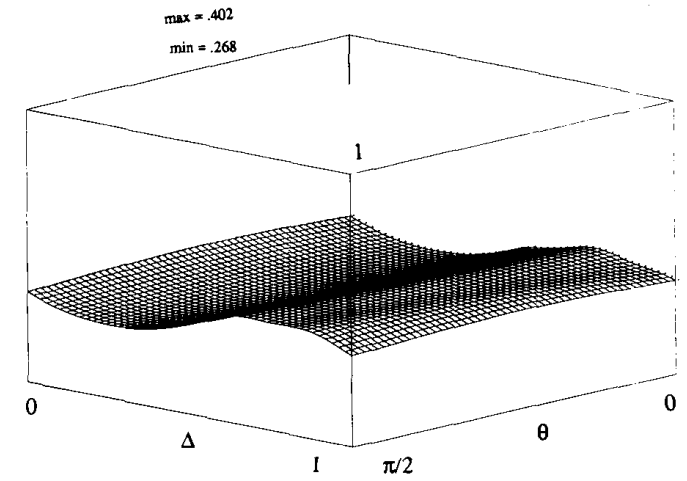

Fig. 8. Noise variance as a function of the starting spreading code phase $\Delta$ and the carrier phase $\theta$ for normalized frequency offsets $\alpha=0.5$, and $\gamma=1$.

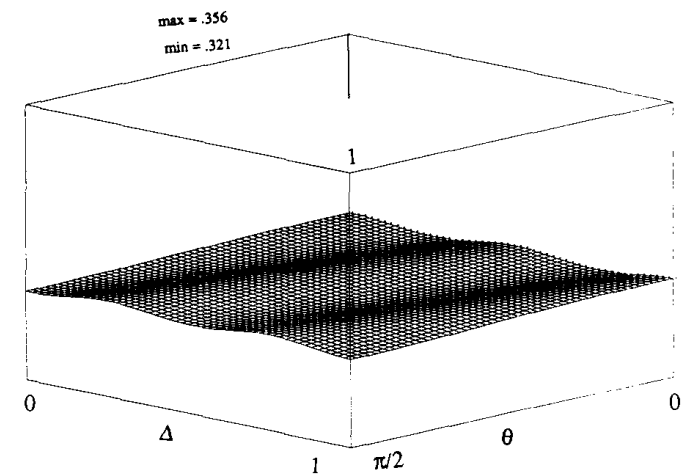

Fig. 9. Noise variànce as a function of the starting spreading code phase $\Delta$ and the carrier phase $\theta$ for normalized frequency offsets $\alpha=1$, and $\gamma$ $=1$.

following three functions:

$$
\begin{aligned}
\nu_{1}(\alpha, \Delta)= & \frac{\lfloor\alpha-\Delta\rfloor}{3 \alpha}+\frac{1}{\alpha}\left(\Delta-\Delta^{2}+\frac{2}{3} \Delta^{3}\right. \\
& \left.+r(\alpha, \Delta)-r^{2}(\alpha, \Delta)+\frac{2}{3} r^{3}(\alpha, \Delta)\right)
\end{aligned}
$$

where $r(\alpha, \Delta)=(\alpha-\Delta)-\lfloor\alpha-\Delta\rfloor$, and $\lfloor x\rfloor$ is equal to the greatest integer less than or equal to $x$.

$$
\begin{aligned}
\nu_{2,1}(\alpha, \Delta)= & 1-\frac{1}{\alpha}\left(\Delta^{2}-(\Delta-\alpha)^{2}-\frac{2}{3} \Delta^{3}\right. \\
& \left.+\frac{2}{3}(\Delta-\alpha)^{3}\right) \\
\nu_{2,2}= & \frac{1}{\alpha}\left(\alpha-\Delta^{2}+\frac{2}{3} \Delta^{3}-(\alpha-\Delta)^{2}\right. \\
& \left.+\frac{2}{3}(\alpha-\Delta)^{3}\right) .
\end{aligned}
$$

We may then write the integral in (9) as

$$
\begin{aligned}
& V(\alpha, 0 ; \Delta, 0) \\
& \quad \begin{cases}\nu_{1}(\alpha, 1-\Delta) & \alpha>1 \\
\nu_{2,1}(\alpha, 1-\Delta) & \alpha \leq 1, \alpha+\Delta \leq 1 \\
\nu_{2,2}(\alpha, 1-\Delta) & \alpha \leq 1, \alpha+\Delta>1 .\end{cases}
\end{aligned}
$$

We would like to find the maximum and minimum of the function $V(\alpha, 0 ; \Delta, 0)$ over the starting phase $\Delta$ so that we may obtain the variation as a function of the offset $\alpha$. To this end we consider the two cases, $\alpha>1$ and $\alpha$ $\leq 1$. For $\alpha>1$ we set $\partial v_{1}(\alpha, \Delta) / \partial \Delta$ equal to zero and solve numerically for the location of the maximum and minimum values. For $\alpha<1$ we can obtain a solution in closed form. For $v_{2.1}(\alpha, \Delta)$ the derivative is equal to zero at $\Delta=(1-\alpha) / 2$ where a minimum of $1 / 2+\alpha^{2} / 6$ is attained. For $v_{2,2}(\alpha, \Delta)$ the derivative is zero at $\alpha / 2$ where a maximum of $1-\alpha / 2+\alpha^{2} / 6$ is attained. We summarized all of these results in Fig. 10 where we have plotted the maximum and minimum values of the variance (in the case that $\theta=0$ ) as a function of the normalized offset clock frequency of the two spreading codes. As expected for $\alpha=0$ there is a lot of variation in the noise variance depending on the (static) clock phases that we may hit upon. As the normalized offset clock frequency increases the variation from the maximum to minimum decreases to zero at $\alpha=1$ and as $\alpha$ increases further some side lobes result with decreasing difference between the maximum and the minimum. For large values of the frequency offset the variance is not dependent on the starting phase. If we take the location of first equality between the maximum and minimum as the point beyond which we have satisfactory variation, then this translates into a required offset frequency between the two spreading code clocks that is greater than the data rate.

\section{B. Zero Spreading Code Frequency Offset}

The opposite case to the above is now considered. If we set the spreading code offset equal to zero $(\alpha=0)$ then the variance is dependent on the starting phase $\Delta$ only through a multiplicative constant. We thus assume that $\Delta$ $=0$ (i.e., the constant equals 1 ). Performing the simplified integral, we obtain

$$
V(0, \gamma ; 0, \theta)=\frac{1}{2}+\frac{1}{8 \pi \gamma}(\sin (4 \pi \gamma+\theta)-\sin (\theta)) \text {. }
$$

We may find the maximum and minimum of (13) over the variable $\theta$ so that we obtain a variation versus the offset frequency $\gamma$. The stationary points are obtained by solving the following equation:

$$
\tan (\theta)=\left(\frac{\cos (4 \pi \gamma)-1}{\sin (4 \pi \gamma)}\right) .
$$

These values may be substituted back in (13) to obtain the maximum and the minimum as a function of the parameter $\gamma$. We have plotted the resulting envelopes in Fig. 11 . The points of zero variation occur at $\gamma$ equal to integer multiples of $1 / 2$. The first point occurs at $1 / 2$ and the first sidelobe has a variation of approximately $(0.4-0.6)$. The second sidelobe falls within the range of (0.43-0.57). The curves converge to $1 / 2$ as expected, however we must keep in mind that our approximation does not hold for large values of $\gamma$.

\section{Joint Frequency Offsets}

Considering $\Delta$ and $\theta$ as random variables we would like to obtain envelopes for the maximum and minimum 


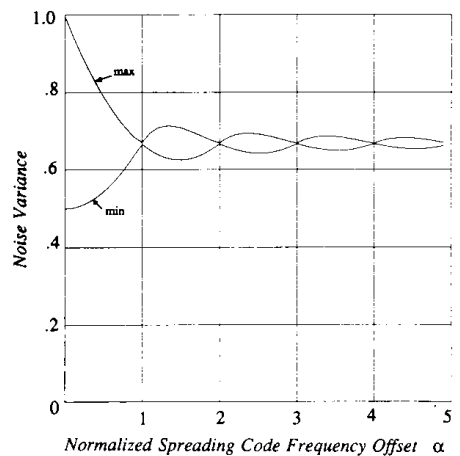

Fig. 10. Maximum and minimum envelopes for the variance over all starting spreading code chip phases $(\Delta)$ versus the normalized spreading code frequency offset $\alpha$ for the case of zero carrier phase and zero offset carrier frequency $(\theta=0, \nu=0)$.

variance over the set of frequency offset pairs $(\alpha, \gamma)$. Such a result is difficult to obtain in closed form. We have however used (5) in an exhaustive computation which we estimate to be accurate to within $5 \%$. We have shown the plot for the maximum as a three-dimensional plot in Fig. 12. From the figure we can see the previous cross-section behavior where the points of zero variation (the minima in this plot) occur at integer multiples along the $\alpha$ axis $(\gamma$ $=0$ ) and at half integer multiples along the $\gamma$ axis ( $\alpha=$ 0 ). However, with joint frequency offsets there are points where the variation is surprisingly large even though the individual variations are zero, e.g. $(\alpha=2, \gamma=1)$ where the variation is approximately $(0.28-0.4)$. The worst points occur along the line where $\alpha=2 \gamma$. If we avoid this region and if the normalized offsets are greater than 1 then the maximum variance can be kept well below the value 0.4 . A constraint on the maximum variance to 0.4 represents a reduction on the maximum variance by approximately $4 \mathrm{~dB}$ when compared to the case of zero offsets, and we are only $0.79 \mathrm{~dB}$ away from the ideal case of $1 / 3$. With some offsets it is possible to constrain the maximum variance to less than 0.35 or $0.21 \mathrm{~dB}$ away from the ideal.

We would like to convert the above noise variance measures, such as in Fig. 12, into probability of error. This however requires that the probability density function for the noise variance be obtained, with $\alpha$ and $\gamma$ as parameters. Such a task is computationally intensive; however, for the case of symbol error probability we can readily obtain upper bounds. For the case of constant variance the probability of symbol error is $\frac{1}{2}$ erfc $(\sqrt{\eta})$ which is shown as curve 1 in Fig. 13; this is our baseline performance curve (the SNR is $\eta$ ). For the case where the variance is random and is given by (8) the probability of error is $\boldsymbol{E}\left(\frac{1}{2}\right.$ erfc $\left.(\sqrt{\eta / 3 V})\right)$ where the expectation is over the variables $\Delta$ and $\theta$. It is plotted as curve 3 in Fig. 13. Now, to obtain an upper bound for the symbol error probability in the case of frequency offsets, let us assume that the offsets are such that the noise variance is always less than 0.4 . We may then assume that the probability density function for the noise variance is a delta function located

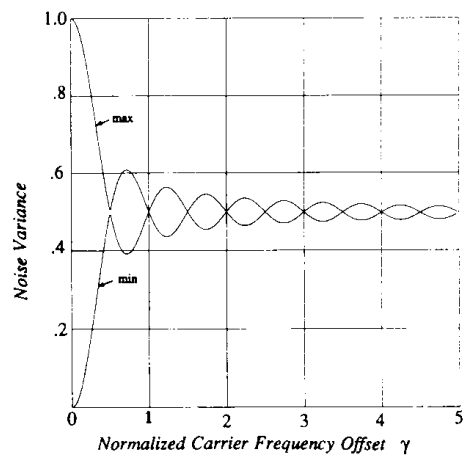

Fig. 11. Maximum and minimum envelopes for the variance over all starting carrier phases $(\theta)$ versus the normalized carrier frequency $\gamma$ for the case for zero spreading code chip phase and zero offset spreading code frequency $(\Delta=0, \alpha=0)$.

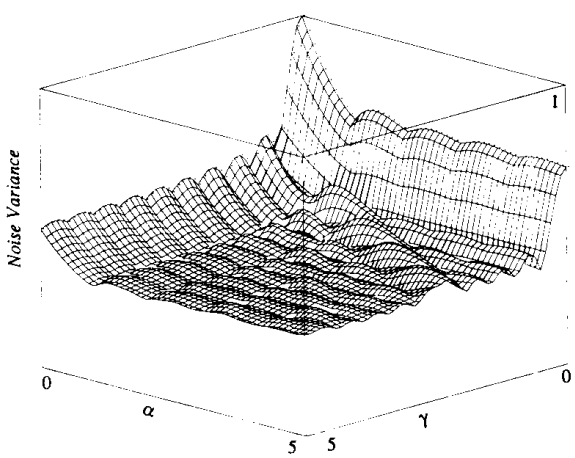

Fig. 12. Maximum noise variance over all starting carrier and spreading code phases as a function of the normalized frequency offsets $\alpha$ and $\gamma$.

at 0.4 ; this is greater than the ideal case of $1 / 3$ by the factor $1 \cdot 2$, thus a bound on the probability of error is $\frac{1}{2}$ $\operatorname{erfc}(\sqrt{\eta / 1.2})$ which is shown as curve 2 in Fig. 13. From the figure we see that at a probability of error of $10^{-3}$ the difference in performance with frequency offsets is greater than $1 \mathrm{~dB}$. For large average SNR the probability of error is dominated by the maximum noise variance and the difference in performance approaches $4 \mathrm{~dB}$.

\section{System Considerations and Conclusions}

As we can see from the above it is beneficial to have frequency offsets between the spreading codes and the carrier frequencies of signals in a multiple-access network utilizing DS/BPSK signaling. A normalized offset equal to or greater than the data rate brings down considerably the variation of the noise variance due to unpredictable starting code and carrier phases. As a result, we propose that in such a network the clocks should be set so that a minimum offset is guaranteed. We do not want to make the offset very large, since for a large number of signals the total frequency band occupied would be considerably larger, hence resulting in loss of bandwidth efficiency. If the network is such that the nodes are stationary then an offset selection scheme, such as those used in channel allocation in a cellular radio network, could be devised to maximize the frequency offset between signals whose 


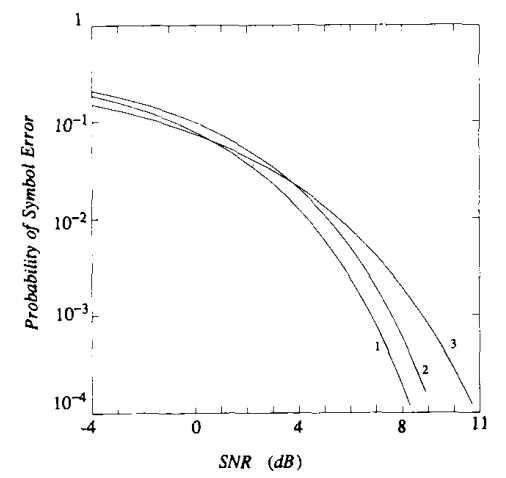

Fig. 13. Probability of symbol error versus average SNR for the cases: 1 ) Gaussian noise, 2) bound assuming variance factor $<0.4,3$ ) no frequency offsets.

transmitters are close to each other. It is only for protection against strong signals that the frequency offset scheme is necessary.

The introduction of an offset into the spreading code clock frequency could have the negative effect of making it slightly more difficult to do signal acquisition. If this is the case then the transmitter could be designed so that the spreading code clock rate is set to a common value at the start of packet transmission and then slowly drifts away from the initial value up to a maximum offset. The receiver code tracking circuits could easily track a small spreading code clock drift.

In a real system frequency offsets may be due to clock drifts resulting from nonstable oscillators and inherent offsets in oscillator crystals, i.e., unintentional offsets; or they may be due to offsets in the oscillators that are imposed in the design, i.e., intentional offsets. The amount of offset in the first case is dependent on the absolute carrier and spreading code frequencies. For a given symbol rate the larger the absolute frequencies are the more difficult it is to keep the unintentional normalized offset frequencies small. Thus, since the spreading code rate is much smaller than the carrier frequency, unintentional offsets are much more likely in the carrier frequencies. Carrier frequency offsets are also susceptible to Doppler shifts which may or may not be significant depending on the data rate. Carrier frequencies are typically in the $\mathrm{GHz}$ range, spreading code clock rates are typically in the $\mathrm{MHz}$ range, and symbol rates are typically in the $\mathrm{KHz}$ range. Thus, it is apparent that the spreading code offsets are controllable and may be designed to be close to an integer multiple of the data rate. Depending on the implementation and on the environment, carrier offsets may not be controllable. In this case we can still force a minimum offset between any two carrier frequency oscillators so as to bound the variation of the variance by one of the sidelobes of the surface in Fig. 12.

In a conventional direct-sequence multiple-access system the probability of symbol and packet error is dependent on the carrier and spreading code chip phases of the interfering signals relative to those of the signal being received. This dependence causes the probability of error, when expressed as a function of the average signal-tonoise ratio, to be greater than the corresponding probability of error for Gaussian noise if the SNR is not to low. We have shown in this paper that this non-Gaussian behavior, which results in poorer performance, can be changed to a "near" Gaussian behavior by imposing an offset frequency between the carrier and spreading code frequencies of the various signals. In the worst case of no carrier and frequency offset the normalized noise variance may be anywhere in the range $(0-1)$ and has expected value equal to $1 / 3$. If the ratios of the difference in carrier frequency to the data rate and the difference in spreading code clock rate to the data rate are greater than 1 then the variation in the normalized variance decreases so that the maximum variance is less than 0.4 . This represents a reduction on the maximum noise variance by approximately $4 \mathrm{~dB}$. Diminishing improvements in the variation of the noise variance are possible by making the normalized frequency offsets greater.

\section{REFERENCES}

[1] M. B. Pursiey, "Performance evaluation of phase-coded spread-spectrum multiple-access communication-Part I: System analysis," IEEE Trans. Commun., vol. COM-25, pp. 795-799, Aug. 1977.

[2] J. S. Lenhart and M. B. Pursley, "Error probabilities for binary directsequence spread-spectrum communications with random signature sequences," IEEE Trans. Commun.. vol. COM-35, pp. 87-98, Jan. 1987.

[3] N. Nazari and R. E. Ziemer, "Computationally efficient bounds for the performance of direct-sequence spread-spectrum multiple-access communications systems in jamming environments," IEEE Trans. Commun., vol. COM-36, pp. 577-587, May 1988.

[4] C. L. Weber, G. K. Huth, and B. H. Batson, "Performance considerations of code division multiple-access systems," IEEE Trans. Vehic. Technol., vol. VT-30, pp. 3-9. Feb. 1981.

[5] J. Y. N. Hui, “Throughput analysis for code division multiple accessing of the spread spectrum channel, "IEEE J. Select. Areas Commun. vol. SAC-2, pp. 482-486, July 1984.

16] E. S. Sousa, "Interference modeling in a direct sequence spread spectrum packet radio networks," IEEE Trans. Commun., to be published.

[7] I. F. Blake and J. B. Thomas, "On a class of processes arising in linear estimation theory," IEEE Trans. Inform. Theory, vol. IT-14, pp. 1216, Jan. 1968.

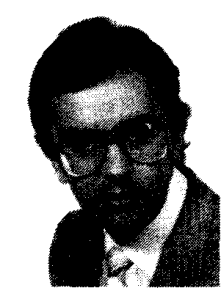

Elvino S. Sousa (S'80-M'86) was born in Azores, Portugal, on December 28, 1956. He received the B.A.Sc. degree in engineering science, the M.A.Sc. degree in electrical engineering from the University of Toronto in 1980 and 1982, respectively, and the $\mathrm{Ph} . \mathrm{D}$. degree in electrical engineering from the University of Southern California in 1985

He was a teaching assistant at the University of Toronto, a research assistant at the University of Southern California, and is currently with the Department of Electrical Engineering at the University of Toronto. He has held a Natural Sciences and Engineering Research Council of Canada (NSERC) Postgraduate Scholarship, was a recipient of the IEEE Communications Society Student Scholarship, and is currently an NSERC University Research Fellow. He has performed research in the areas of data transmission and spread-spectrum packet radio networks. He is also interested in optical communications and on the performance of distributed computer systems where he has consulted for Technology Transfer Institute. In the past three years at the University of Toronto he has taught graduate courses in error-correcting codes and mobile communications.

Dr. Sousa is a member of Eta Kappa Nu and a member of the Association of Professional Engineers of Ontario. 